\title{
Anthocyanin kinetics are dependent on anthocyanin structure
}

\author{
Janet A. Novotny*, Beverly A. Clevidence and Anne C. Kurilich $\dagger$ \\ U. S. Department of Agriculture, Agricultural Research Service, Beltsville Human Nutrition Research Center, Beltsville, \\ MD 20705, USA
}

(Received 5 October 2010 - Revised 14 April 2011 - Accepted 9 May 2011 - First published online 28 September 2011)

\section{Abstract}

The kinetics of anthocyanin metabolism was investigated in a human feeding trial. Volunteers ( $n$ 12) consumed purple carrots containing five anthocyanin forms: cyanidin-3-(xylose-glucose-galactoside), cyanidin-3-(xylose-galactoside), cyanidin-3-(xylose-sinapoyl-glucosegalactoside), cyanidin-3-(xylose-feruloyl-glucose-galactoside) and cyanidin-3-(xylose-coumuroyl-glucose-galactoside). The purple carrots were served as three different treatments in a crossover design with a 3-week washout between treatments. Purple carrot treatments were $250 \mathrm{~g}$ raw carrots, $250 \mathrm{~g}$ cooked carrots and $500 \mathrm{~g}$ cooked carrots. Serial blood and urine samples were collected for 8 and $24 \mathrm{~h}$ after the dose, respectively, and analysed for anthocyanins. Of the anthocyanin forms ingested, four were detected in plasma and urine: cyanidin-3-(xylose-glucose-galactoside), cyanidin-3-(xylose-galactoside), cyanidin-3-(xylose-sinapoyl-glucose-galactoside) and cyanidin-3-(xylose-feruloyl-glucose-galactoside). The time courses of plasma and urine anthocyanin contents were evaluated with compartmental modelling. Results showed that absorption, gastrointestinal transit and plasma elimination are dependent on anthocyanin structure. Absorption efficiencies of acylated compounds (cyanidin-3-(xylose-sinapoyl-glucose-galactoside) and cyanidin-3-(xylose-feruloyl-glucose-galactoside)) were less than those for non-acylated anthocyanins (cyanidin-3-(xylose-glucose-galactoside) and cyanidin-3(xylose-galactoside)). The acylated anthocyanins exhibited a shorter half-life for gastrointestinal absorption than the non-acylated anthocyanins. Fractional elimination of non-acylated compounds was slower than that for acylated anthocyanins. These results provide the first information about the kinetics of individual anthocyanins in human beings.

Key words: Mathematical modelling: Cyanidins: Kinetics: Flavonoids

Anthocyanins are a class of highly pigmented flavonoids distributed throughout nature. In addition to imparting red, purple and blue colour to many fruits and vegetables in our diet, anthocyanins have been associated with a variety of health benefits, including reducing risk factors of CVD in both human ${ }^{(1,2)}$ and animal ${ }^{(3-6)}$ models, protecting cognitive function in both human ${ }^{(7)}$ and animal ${ }^{(8,9)}$ models and improving glucose metabolism ${ }^{(10-12)}$. To maximise the benefit of anthocyanins in the diet, it is important to understand the bioavailability and metabolism of these compounds.

Previous studies have shown that many factors affect bioavailability. Several studies have shown that anthocyanins exhibit a complex dose response, with decreasing absorption efficiencies at increasing dose levels ${ }^{(13-15)}$. Acylation results in a substantial decline in bioavailability ${ }^{(13-15)}$. Anthocyanin backbone is also a key determinant of bioavailability, with pelargonidin-based anthocyanins being absorbed with much greater efficiencies than anthocyanins with other backbones ${ }^{(16,17)}$. Metabolism is also profoundly affected by anthocyanin backbone, with pelargonidin-based compounds being primarily converted to metabolites, while a larger proportion of compounds with other backbones appearing intact after absorption ${ }^{(16,17)}$.

While structure has been shown to have a profound impact on absorption and conversion to metabolites ${ }^{(14,16,17)}$, very little is known about the effect of anthocyanin structure on anthocyanin pharmacokinetics. The objective of the present study was to determine the differences in pharmacokinetics (absorption, distribution, metabolism and excretion) of four different cyanidin-based anthocyanins. The effect of cooking on anthocyanin absorption was also investigated, since cooking has been shown to influence phytonutrient absorption $^{(18)}$. Understanding of differences in absorption and metabolism rates of anthocyanins will be useful for development of intake recommendations for optimising health and reducing risk of chronic disease.

Abbreviations: GI, gastrointestinal; TFA, trifluoroacetic acid.

*Corresponding author: J. A. Novotny, fax +301504 9098, email janet.novotny@ars.usda.gov

†Present address: PepsiCo, Barrington, IL 60 010, USA. 


\section{Experimental methods}

Subjects, study design, diet and sample collection

A total of twelve non-smoking, healthy adults (six males and six females) from the Beltsville, MD, area participated in the present study. The study was conducted according to the guidelines laid down in the Declaration of Helsinki, and all procedures involving human subjects were approved by the Johns Hopkins University Bloomberg School of Public Health Committee on Human Research. Written informed consent was obtained from all subjects. Subject characteristics were 43 (SD 13) years old, 72 (SD 14) $\mathrm{kg}$ and BMI of 24 (SD 3.4) kg/m².

The study was conducted as a three-treatment crossover with periods separated by 3-week breaks. Subjects were randomly assigned to one of the three groups ( $n$ 4), with each group receiving a different treatment order. Treatments were as following: $250 \mathrm{~g}$ purple carrot sticks served raw, $250 \mathrm{~g}$ purple carrot sticks served microwave-cooked and $500 \mathrm{~g}$ purple carrot sticks served microwave-cooked. Subjects consumed carrot treatments after a $12 \mathrm{~h}$ fast and after $2 \mathrm{~d}$ of an anthocyanin-free diet. Raw carrots were consumed with $6 \mathrm{~g}$ fat as dressing (soyabean and rapeseed oil) and cooked carrots were consumed with $6 \mathrm{~g}$ butter.

The purple carrots were washed, cut into sticks with the orange core discarded and mixed for batch uniformity. For cooked treatments, carrots were weighed before cooking and then one tablespoon or three tablespoons of water was added to 250 and $500 \mathrm{~g}$ doses. Cooked treatments were microwave-cooked (900 W microwave ovens; Sharp Electronics Corporation, Mahwah, NJ, USA) while covered for 12 or $20 \mathrm{~min}$ for the 250 and $500 \mathrm{~g}$ doses. For each treatment period, samples of cooked and raw carrots were frozen at $-80^{\circ} \mathrm{C}$ until extraction and analysis.

Blood was collected at $0,0 \cdot 5,1,1 \cdot 5,2,2 \cdot 5,3,3 \cdot 5,4,5,6,7$ and $8 \mathrm{~h}$. Urine samples were collected at baseline and every $2 \mathrm{~h}$ until $16 \mathrm{~h}$. From 16 to $24 \mathrm{~h}$ urine was pooled. During the collection period, subjects were provided an anthocyaninfree diet that included a snack, lunch and dinner provided at 2, 4 and $10 \mathrm{~h}$, respectively, after carrot consumption. Caffeine consumption was prohibited on the dose day and for $1 \mathrm{~d}$ before dose. Vitamins and supplements were prohibited throughout the study.

\section{Reagents}

Cyanidin-3-galactoside and malvidin-3-galactoside were purchased from Indofine Chemical Company (Somerville, NJ, USA). Ethyl acetate and HPLC grade water and methanol were obtained from Thermo Fisher Scientific, Inc. (Pittsburgh, PA, USA). Formic acid and trifluoroacetic acid (TFA) were obtained from Sigma-Aldrich (St Louis, MO, USA).

\section{Sample preparation for analysis}

Carrots $(60 \mathrm{~g})$ were ground in a Waring blender (Torrington, CT, USA) with liquid $\mathrm{N}_{2}$ to form a powder. Powdered carrots (duplicate $3 \mathrm{~g}$ samples) were weighed into a test tube before adding $10 \mathrm{ml}$ of $10 \%$ methanolic formic acid. Samples were vortex-mixed for $1 \mathrm{~min}$, sonicated for $10 \mathrm{~min}$ and then centrifuged at $2500 \mathrm{~g}$ for $10 \mathrm{~min}$. The supernatant was decanted to a collection vial and samples were extracted three more times with $10 \mathrm{ml}$ of $10 \%$ methanolic formic acid. The combined extract was diluted $10 \times$ with methanol-10\% aqueous formic acid (1:9) before injecting onto the liquid chromatography-MS

Blood samples were collected into vacutainers containing EDTA and centrifuged at $2560 \mathrm{~g}$ for $10 \mathrm{~min}$. Aqueous TFA $(1.3 \mathrm{ml}$ of $0.44 \mathrm{M})$ was added to plasma aliquots $(2.2 \mathrm{ml})$ and urine was weighed and $10 \mathrm{ml}$ aliquots were stored in vials containing $2 \mathrm{ml}$ of $0.44 \mathrm{M}$-TFA. All samples were stored at $-80^{\circ} \mathrm{C}$ until analysis.

Before HPLC quantification of anthocyanins in plasma and urine, samples were thawed and malvidin-3-galactoside was added as an internal standard. Isolation and concentration of anthocyanins was performed using solid-phase extraction. SPE C-18 Sep-Pak cartridges (Waters Corporation, Milford, MA, USA) were conditioned with $5 \mathrm{ml}$ methanol and then $5 \mathrm{ml}$ of $0.44 \mathrm{M}$ aqueous TFA before adding the sample. After adding the sample, water-soluble compounds were eluted with $5 \mathrm{ml}$ of $0.44 \mathrm{~m}$ aqueous TFA, followed by elution of polar non-anthocyanin phenolics with $5 \mathrm{ml}$ ethyl acetate before removing and collecting anthocyanins with $5 \mathrm{ml}$ of $0.44 \mathrm{M}$ methanolic TFA. Samples were dried under $\mathrm{N}_{2}$ and then reconstituted in methanol-10\% aqueous formic acid (1:9).

\section{Liquid chromatography-MS conditions}

The liquid chromatography-MS instrument was composed of an Agilent HP series 1100 G1946A mass selective detector with an electrospray ionisation source connected to a G1315A diode array detector, temperature-controlled column compartment $\left(30^{\circ} \mathrm{C}\right)$, autosampler, automatic solvent degasser, binary pump, Zorbax SB-C18 column $(5 \mu \mathrm{m}, 250 \times 4.6 \mathrm{~mm})$ and guard cartridge (Agilent Technologies, Wilmington, DE, USA). The solvent system consisted of $10 \%$ aqueous formic acid (solvent A) and methanol (solvent B). The gradient was linear from $5 \%$ solvent B to $55 \%$ solvent B over 20 min, then $100 \%$ solvent B for $5 \mathrm{~min}$ to flush the column and back to $5 \%$ solvent $\mathrm{B}$ for $10 \mathrm{~min}$ to re-equilibrate the column. The flow rate was $1 \mathrm{ml} /$ min and injection volume was $50 \mu$ l. The mass selective detector source was positive electrospray ionisation with the spray chamber gas temperature set at $300^{\circ} \mathrm{C}$, nebuliser pressure set at $60 \mathrm{psig}$, VCap at $3000 \mathrm{~V}$ and drying gas $\left(\mathrm{N}_{2}\right)$ at 12 litres $/ \mathrm{min}$. Selected ion monitoring was used to identify individual anthocyanins and metabolites in the carrot, plasma and urine samples and to monitor potential anthocyanin glucuronide or sulphate metabolites. The G1315A diode array detector was set to collect signal at $530 \mathrm{~nm}$. Limit of spectral detection for cyanidin3-galactoside (Cy-3-gal; external standard for quantification) was $0.025 \mathrm{nmol} / \mathrm{ml}$ for a $50 \mu \mathrm{l}$ injection. Data were collected using Agilent ChemStation software (Wilmington, DE, USA) and quantified from the G1315A diode array detector.

\section{Calculations, statistics and compartmental modelling}

The internal standard malvidin-3-galactoside was used to account for sample loss during extraction, which was on 
Table 1. Anthocyanin contents ( $\mu \mathrm{mol})$ of treatments

\begin{tabular}{lcccccc}
\hline & Total & Су3XG & Су3XGG & Су3XSGG & Cy3XFGG & Cy3XCGG \\
\hline Raw carrots $(250 \mathrm{~g})$ & 463 & 36 & 27 & 263 & 129 & 8 \\
Cooked carrots $(250 \mathrm{~g})$ & 357 & 27 & 22 & 215 & 88 & 6 \\
Cooked carrots $(500 \mathrm{~g})$ & 714 & 53 & 44 & 430 & 177 & 11 \\
\hline
\end{tabular}

СузXG, cyanidin-3-(xylose-galactoside); СузXGG, cyanidin-3-(xylose-glucose-galactoside); Cy3XSGG, cyanidin-3-(xylosesinapoyl-glucose-galactoside); Cy3XFGG, cyanidin-3-(xylose-feruloyl-glucose-galactoside); Cy3XCGG, cyanidin-3-(xylosecoumuroyl-glucose-galactoside).

average $25 \%$. Molar concentrations of individual anthocyanins were calculated using an external standard curve of Cy-3-gal, since standards for the anthocyanins monitored were not available.

The WinSAAM software package (version 3.0.7; Kennett Square, PA, USA) was used to develop a compartmental model of anthocyanin kinetics. The mathematical prediction of plasma and urine anthocyanin contents for each anthocyanin was compared to measured data points and model parameters were adjusted until model prediction was in good accord with observed data. A least-squares procedure was then used to minimise the difference between model prediction and observed data.

Model parameter values across treatments were compared using a one-way repeated-measures ANOVA, and pair-wise comparisons were carried out with a Tukey's test. $P$ values $<0.05$ were considered statistically different. Statistics were performed using SigmaStat software (version 3.0; Aspire Software International, Ashburn, VA, USA).

\section{Results and discussion}

Five anthocyanins forms were present in the carrot treatments: cyanidin-3-(xylose-glucose-galactoside), cyanidin-3(xylose-galactoside), cyanidin-3-(xylose-sinapoyl-glucose-galactoside), cyanidin-3-(xylose-feruloyl-glucose-galactoside) and cyanidin-3-(xylose-coumuroyl-glucose-galactoside). Total anthocyanin content of treatments ranged from 357 to $714 \mu \mathrm{mol}$ (Table 1). Content of individual anthocyanins ranged from 6 to $430 \mu \mathrm{mol}$. All anthocyanin forms except cyanidin-3-(xylosecoumuroyl-glucose-galactoside) were detected in both plasma and urine (Fig. 1).

A twelve-compartment model (Fig. 2), composed of four sub-models (one for each anthocyanin form) of three compartments each, was used to analyse the kinetics of the individual anthocyanins in purple carrots. Each sub-model consisted of an upper gastrointestinal (GI) compartment, a plasma compartment and a urine compartment. Movement of anthocyanins through the system was described by firstorder linear equations. The parameters $\mathrm{K}_{j, i}$ represent fractional transfer coefficients, describing the fraction of material in compartment $i$ that is transferred to compartment $j$ per unit time (with subscript $\mathrm{K}_{O, i}$ representing movement out of the system). Upper GI passage and absorption were calculated as separate parameters, and a multiplicative function of the two described the movement from the GI tract into plasma. Fractional SD on all model parameters after least-squares minimisation of residuals were less than $10 \%$ (fractional SD of up to $60 \%$ are considered acceptable to conclude that actual parameter values have been identified $\left.{ }^{(19)}\right)$. The model allowed determination of absorption efficiencies, rates and half-lives for upper GI transfer into plasma and rates and half-lives for plasma elimination of anthocyanins into urine. Values for absorption efficiencies (mass absorbed divided by
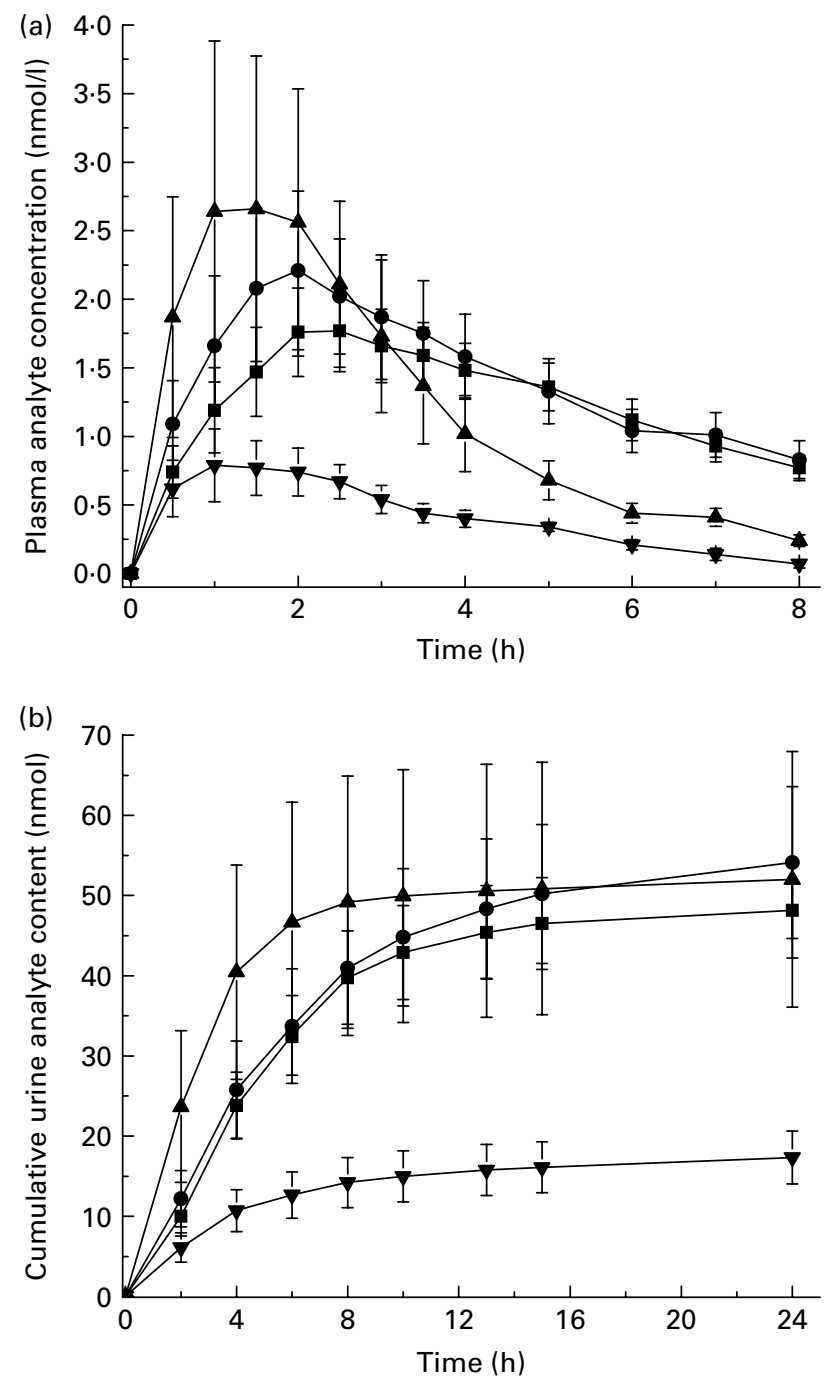

Fig. 1. Plasma anthocyanin concentration (a) and cumulative urinary anthocyanin content (b) after volunteers consumed $250 \mathrm{~g}$ raw purple carrots. Values represent means with their standard errors. Cy3XG, cyanidin-3-(xylose-galactoside) (- - ); СузXGG, cyanidin-3-(xylose-glucose-galactoside) (—-); Cy3XSGG, cyanidin-3-(xylose-sinapoyl-glucose-galactoside) ( - - $)$; Cy3XFGG, cyanidin-3-(xylose-feruloyl-glucose-galactoside) ( $\longrightarrow \boldsymbol{\nabla})$. 


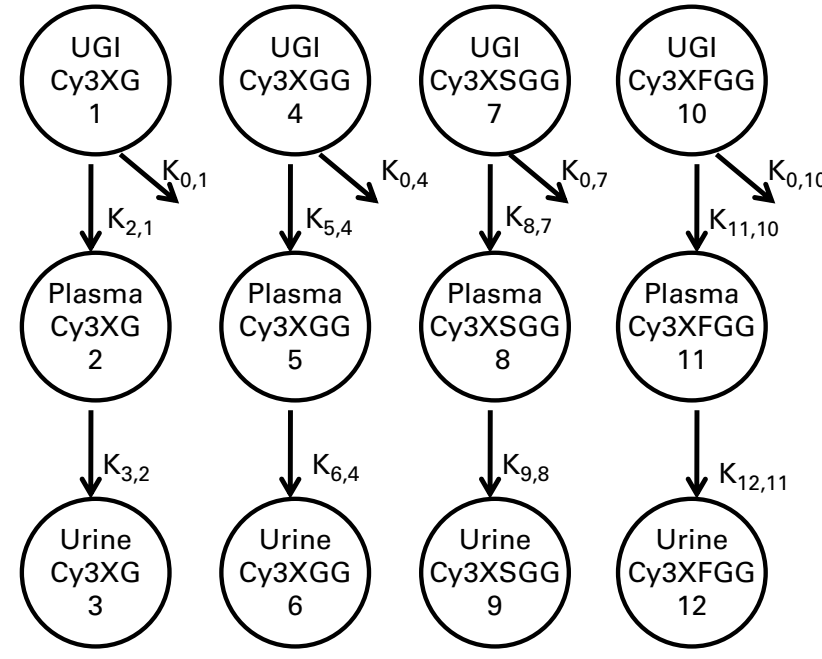

Fig. 2. Compartmental model of anthocyanin metabolism. $\mathrm{K}_{i, j}$ represents the fractional transfer coefficient from compartment $j$ to compartment $i$. For irreversible exit from the system, the fractional transfer coefficient is referred to as $\mathrm{K}_{o, j}$. UGI, upper gastrointestinal tract; Cy3XG, cyanidin-3(xylose-galactoside); Cy3XGG, cyanidin-3-(xylose-glucose-galactoside); Cy3XSGG, cyanidin-3-(xylose-sinapoyl-glucose-galactoside); Cy3XFGG, cyanidin-3-(xylose-feruloyl-glucose-galactoside).

mass of dose expressed as percentage) for individual treatments were calculated separately, while values for rates and half-lives of upper GI transfer into plasma and for plasma elimination of anthocyanins into urine were calculated from pooled data because they did not show differences by treatment.

Cooking did not have an impact on bioavailability of anthocyanins (Table 2). In contrast, a decrease in absorption efficiency of individual anthocyanins was observed with the larger dose. When the data were pooled to calculate absorption of total anthocyanins ${ }^{(13)}$, the absorption efficiencies for the larger and smaller doses were not significantly different; presumably the difference was lost due to the increased variability resulting from pooling the data for individual anthocyanins (Table 3).

Absorption efficiency of individual anthocyanins ranged from 0.12 to $0 \cdot 25 \%$ for non-acylated anthocyanins and from 0.0079 to $0.019 \%$ for acylated anthocyanins. For each treatment, absorption efficiencies of the two acylated anthocyanins did not differ from each other and absorption efficiencies of the two non-acylated anthocyanins did not differ from each other, but non-acylated anthocyanins were absorbed with greater efficiencies than acylated anthocyanins. This may be partially related to the lower affinity of acylated anthocyanins for transporter bilitranslocase. Bilitranslocase is an organic anion carrier found in tissues, including liver ${ }^{(20)}$, gastric musoca $^{(21)}$ and kidney ${ }^{(22)}$. A previously published study of the interaction between anthocyanins and bilitranslocase suggests that mono- and di-glucosylated anthocyanins were good ligands for bilitranslocase, whereas the acylated anthocyanins that were tested appeared not to be ${ }^{(23)}$ good ligands for bilitranslocase.

Based on plasma appearance, rates of passage through the upper GI tract showed half-lives ranging from $2 \cdot 2$ to $8 \mathrm{~h}$. This may seem longer than expected, since the peak plasma concentration was observed within the first few hours, and since previous studies have also shown very early peak plasma times for anthocyanins, as reviewed by McGhie \& Walton ${ }^{(24)}$. However, these anthocyanins were delivered embedded in the carrot matrix, thus the GI transit time reflects time for digestion and release of anthocyanins from the carrot tissue as well as absorption. Early peak plasma times, therefore, would reflect easily accessible anthocyanins and digestion time would be extended as anthocyanins are released from the plant matrix. The acylated anthocyanins exhibited shorter half-lives for GI absorption than the non-acylated. This may reflect the absorbability of non-acylated anthocyanins along a larger proportion of the GI tract. In other words, if acylated anthocyanins are absorbed across a shorter length of the GI tract, then the effective half-life of transit into plasma through the absorbing section of the GI tract would be reduced. Cyanidin-3-(xylose-sinapoyl-glucose-galactoside had the shortest upper GI transfer time, followed by cyanidin-3-(xylose-feruloyl-glucose-galactoside), then the two non-acylated anthocyanins, for which upper GI transfer time was not different.

Elimination of different anthocyanins from the plasma was also dependent on structure. Non-acylated compounds were eliminated more slowly than acylated compounds. Elimination rates of the two non-acylated compounds did not differ from each other and those of the two acylated compounds did not differ from each other. As with GI absorption, differences in interactions with transporters at the tissue level (such as bilitranslocase which is found in liver ${ }^{(20)}$, kidney $^{(21)}$ and vascular epithelium ${ }^{(25)}$ ) may explain the differences in the observed plasma kinetics of the different anthocyanin forms.

Many studies have shown that anthocyanins are absorbed and excreted intact. Possible metabolites include glucuronidated,

Table 2. Absorption efficiency (\%) of anthocyanins for different carrot treatments

(Mean values with their standard errors)

\begin{tabular}{|c|c|c|c|c|c|c|c|c|}
\hline & \multicolumn{2}{|c|}{ СузXG } & \multicolumn{2}{|c|}{ СузXGG } & \multicolumn{2}{|c|}{ Сy3XSGG } & \multicolumn{2}{|c|}{ СузXFGG } \\
\hline & Mean & SEM & Mean & SEM & Mean & SEM & Mean & SEM \\
\hline Raw carrots $(250 \mathrm{~g})$ & $0 \cdot 16^{\mathrm{a}, \mathrm{c}, \mathrm{d}}$ & 0.03 & $0.25^{a}$ & 0.04 & $0.019^{b, c}$ & 0.006 & $0.013^{b, c, d}$ & 0.003 \\
\hline Cooked carrots $(250 \mathrm{~g})$ & $0.19^{\mathrm{a}, \mathrm{c}}$ & 0.02 & $0.23^{\mathrm{a}}$ & 0.04 & $0.013^{b, c, d}$ & 0.001 & $0.013^{b, c}$ & 0.001 \\
\hline Cooked carrots $(500 \mathrm{~g})$ & $0 \cdot 12^{\mathrm{a}, \mathrm{d}}$ & 0.02 & $0.16^{a}$ & 0.02 & $0.0079^{b, d}$ & 0.0007 & $0.0090^{\mathrm{b}, \mathrm{d}}$ & 0.0008 \\
\hline
\end{tabular}

СузXG, cyanidin-3-(xylose-galactoside); СузXGG, cyanidin-3-(xylose-glucose-galactoside); Cy3XSGG, cyanidin-3-(xylose-sinapoyl-glucose-galactoside); Сy3XFGG, cyanidin-3-(xylose-feryloyl-glucose-galactoside).

${ }^{a, b}$ Mean values were statistically different between anthocyanin forms $(P<0.05)$.

${ }^{\mathrm{c}, \mathrm{d}}$ Mean values were statistically different between treatments $(P<0.05)$. 
Table 3. Kinetic parameters of individual anthocyanin-based compounds

(Mean values with their standard errors)

\begin{tabular}{|c|c|c|c|c|c|c|c|c|}
\hline & \multicolumn{2}{|c|}{ СузXG } & \multicolumn{2}{|c|}{ СузXGG } & \multicolumn{2}{|c|}{ Cy3SXGG } & \multicolumn{2}{|c|}{ Су3FXGG } \\
\hline & Mean & SEM & Mean & SEM & Mean & SEM & Mean & SEM \\
\hline Rate of upper Gl transfer into plasma (fraction per $\mathrm{h}$ ) & $0 \cdot 18^{\mathrm{a}}$ & 0.02 & $0 \cdot 13^{a}$ & 0.01 & $0.34^{b}$ & 0.02 & $0 \cdot 26^{c}$ & 0.02 \\
\hline Upper GI half-life $(\mathrm{h})$ & $5 \cdot 1^{\mathrm{a}}$ & 0.5 & $7 \cdot 8^{\mathrm{a}}$ & $1 \cdot 3$ & $2 \cdot 2^{\mathrm{b}}$ & $0 \cdot 1$ & $3 \cdot 0^{\mathrm{c}}$ & 0.2 \\
\hline Rate of elimination from plasma (fraction per $\mathrm{h}$ ) & $1 \cdot 14^{\mathrm{a}}$ & 0.05 & $1 \cdot 12^{\mathrm{a}}$ & 0.04 & $1.54^{b}$ & 0.07 & $1 \cdot 34^{b}$ & 0.05 \\
\hline Plasma half-life $(\mathrm{h})$ & $0.64^{\mathrm{a}}$ & 0.02 & $0.65^{\mathrm{a}}$ & 0.02 & $0.48^{\mathrm{b}}$ & 0.02 & $0.54^{\mathrm{b}}$ & 0.02 \\
\hline
\end{tabular}

СузXG, cyanidin-3-(xylose-galactoside); Cy3XGG, cyanidin-3-(xylose-glucose-galactoside); Cy3XSGG, cyanidin-3-(xylose-sinapoyl-glucose-galactoside); Cy3XFGG, cyanidin-3-(xylose-feryloyl-glucose-galactoside); Gl, gastrointestinal.

a,b Mean values were statistically different between anthocyanin forms $(P<0.05)$.

sulphated and/or methylated conjugates ${ }^{(14,17,26-29)}$, and different anthocyanins are metabolised to different extents ${ }^{(27)}$. These metabolites may be formed by enzymes such as UDP-glucuronosyltransferase, UDP-glucose dehydrogenase or catechol$O$-methyltransferase, and these biotransformations may take place in the small intestine, liver or kidney. For cyanidin-based anthocyanins, as studied in the present experiment, many reports have suggested that the intact forms are the primary forms found in blood and urine $e^{(14,26,30,31)}$. For the present experiment, we looked for but did not detect glucuronidated or sulphated metabolites in either blood or urine, suggesting that these anthocyanins were primarily absorbed and excreted intact. The ability to obtain good agreement between model prediction and experimental data with a mathematical model that was based only on intact forms is in accord with minimal metabolism of these complex anthocyanin forms. Protocatechuic acid is a possible degradation product of cyanidin-based anthocyanins ${ }^{(32-35)}$. We did not monitor potential formation of phenolic acids. Protocatechuic acid can be formed from the cyanidin aglycone when exposed to nearly neutral $\mathrm{pH}^{(34,35)}$, possibly in blood, in the colon or during sample processing. The importance of this pathway in metabolism of anthocyanins is unclear ${ }^{(31,36)}$.

The physiological behaviour of the complex cyanidin derivatives in purple carrots was similar to that observed for simpler cyanidin forms. Both chokeberry and elderberry contain primarily simple cyanidin forms and thus offer a useful comparison. Previous studies with chokeberry and elderberry delivered doses as low as $147 \mathrm{mg}$ and as high as $3.57 \mathrm{~g}^{(37-43)}$; the doses used in the present study (321-643 mg total anthocyanins) fell in the centre of this range. We observed a maximum plasma total anthocyanin concentration of $5-5.8 \mathrm{nmol} / 1$, compared to $97 \mathrm{nmol} / \mathrm{l}$ for elderberry extract containing $720 \mathrm{mg}$ cyanidin-based anthocyanins ${ }^{(38)}, 96 \mathrm{nmol} / 1$ for chokeberry extract containing $721 \mathrm{mg}$ cyanidin-based anthocyanins ${ }^{(41)}$ or $32 \mathrm{nmol} / 1$ for chokeberry juice delivering cyanidin-based anthocyanins at $0.8 \mathrm{mg} / \mathrm{kg}$ body weight ${ }^{(43)}$. These comparisons, though limited, suggest that the more complex anthocyanins, especially the acylated anthocyanins, have more limited absorption than the simpler cyanidin forms. Plasma half-lives reported from other studies ${ }^{(31,42,43)}$ tended to be longer (on the order of $2 \mathrm{~h}$ ) than those reported here because previous half-lives were calculated simply from plasma decay curves, which are influenced not only by pathways of anthocyanin exit from plasma but also by simultaneous entry of anthocyanins into plasma. If one does not account for these multiple processes when calculating half-lives, the estimation of half-life is inaccurate (and lengthened when entry into plasma is neglected). Mathematical modelling accounts for the different processes occurring simultaneously, thus delivers more accurate values for half-lives. Our plasma half-life values ranged from 29 to $38 \mathrm{~min}$ for the different anthocyanin forms.

The present study reports for the first time the rates of absorption and elimination for anthocyanins. Differences in rates of metabolism highlight where differences in metabolic pathways and mechanisms probably exist. In addition, information about rates of absorption and elimination of nutrients and phytonutrients provide critical pieces of information for development of intake recommendations.

\section{Acknowledgements}

Author contributions were as follows: J. A. N., B. A. C. and A. C. K. designed and conducted the study; A. C. K. performed the laboratory analysis; and J. A. N. conducted the mathematical modelling, interpreted data, and wrote the manuscript. This work was supported by the USDA Agricultural Research Service and an USDA CSREES IFAFS grant 2000-4258. There are no conflicts of interest to report.

\section{References}

1. McKay DL \& Blumberg JB (2007) Cranberries (Vaccinium macrocarpon) and cardiovascular disease risk factors. Nutr Rev 65, 490-502.

2. Ruel G, Pomerleau S, Couture P, et al. (2008) Low-calorie cranberry juice supplementation reduces plasma oxidized LDL and cell adhesion molecule concentrations in men. Br J Nutr 99, 352-359.

3. Tsuda T, Horio F, Kato Y, et al. (2002) Cyanidin 3-O-betaD-glucoside attenuates the hepatic ischemia-reperfusion injury through a decrease in the neutrophil chemoattractant production in rats. J Nutr Sci Vitaminol (Tokyo) 48, 134-141.

4. Xia X, Ling W, Ma J, et al. (2006) An anthocyanin-rich extract from black rice enhances atherosclerotic plaque stabilization in apolipoprotein E-deficient mice. J Nutr 136, 2220-2225.

5. Toufektsian MC, de Lorgeril M, Nagy N, et al. (2008) Chronic dietary intake of plant-derived anthocyanins protects the rat heart against ischemia-reperfusion injury. $J$ Nutr $\mathbf{1 3 8}$, $747-752$. 
6. Mauray A, Milenkovic D, Besson C, et al. (2009) Atheroprotective effects of bilberry extracts in apo E-deficient mice. J Agric Food Chem 57, 11106-11111.

7. Krikorian R, Shidler MD, Nash TA, et al. (2010) Blueberry supplementation improves memory in older adults. J Agric Food Chem 58, 3996-4000.

8. Wang J, Ho L, Zhao W, et al. (2008) Grape-derived polyphenolics prevent Abeta oligomerization and attenuate cognitive deterioration in a mouse model of Alzheimer's disease. J Neurosci 28, 6388-6392.

9. Joseph JA, Shukitt-Hale B \& Willis LM (2009) Grape juice, berries, and walnuts affect brain aging and behavior. J Nutr 139, 1813S-1817S.

10. Jayaprakasam B, Olson LK, Schutzki RE, et al. (2006) Amelioration of obesity and glucose intolerance in high-fat-fed C57BL/6 mice by anthocyanins and ursolic acid in Cornelian cherry (Cornus mas). J Agric Food Chem 54, 243-248.

11. Prior RL, Wu X, Gu L, et al. (2008) Whole berries versus berry anthocyanins: interactions with dietary fat levels in the C57BL/6J mouse model of obesity. J Agric Food Chem 56, 647-653.

12. Takikawa M, Inoue S, Horio F, et al. (2010) Dietary anthocyanin-rich bilberry extract ameliorates hyperglycemia and insulin sensitivity via activation of AMP-activated protein kinase in diabetic mice. $J$ Nutr 140, 527-533.

13. Kurilich AC, Clevidence BA, Britz SJ, et al. (2005) Plasma and urine responses are lower for acylated $v$ s nonacylated anthocyanins from raw and cooked purple carrots. J Agric Food Chem 53, 6537-6542.

14. Charron CS, Clevidence BA, Britz SJ, et al. (2007) Effect of dose size on bioavailability of acylated and nonacylated anthocyanins from red cabbage (Brassica oleracea L. Var. capitata). J Agric Food Chem 55, 5354-5362.

15. Charron CS, Kurilich AC, Clevidence BA, et al. (2009) Bioavailability of anthocyanins from purple carrot juice: effects of acylation and plant matrix. J Agric Food Chem 57, 1226-1230.

16. Felgines C, Texier O, Besson C, et al. (2007) Strawberry pelargonidin glycosides are excreted in urine as intact glycosides and glucuronidated pelargonidin derivatives in rats. Br J Nutr 6, 1126-1131.

17. Carkeet C, Clevidence BA \& Novotny JA (2008) Anthocyanin excretion by humans increases linearly with increasing strawberry dose. J Nutr 138, 897-902.

18. Fielding JM, Rowley KG, Cooper P, et al. (2005) Increases in plasma lycopene concentration after consumption of tomatoes cooked with olive oil. Asia Pac J Clin Nutr 14, 131-136.

19. Wastney ME, Patterson BH, Linares OA, et al. (1999) Invetigating Biological Systems Using Modeling. Boston: Academic Press.

20. Baldini G, Passamonti S, Lunazzi GC, et al. (1986) Cellular localization of sulfobromophthalein transport activity in rat liver. Biochim Biophys Acta 856, 1-10.

21. Battiston L, Macagno A, Passamonti S, et al. (1999) Specific sequence-directed anti-bilitranslocase antibodies as a tool to detect potentially bilirubin-binding proteins in different tissues of the rat. FEBS Lett 453, 351-355.

22. Vanzo A, Terdoslavich M, Brandoni A, et al. (2008) Uptake of grape anthocyanins into the rat kidney and the involvement of bilitranslocase. Mol Nutr Food Res 52, 1106-1116.

23. Passamonti S, Vrhovsek U \& Mattivi F (2002) The interaction of anthocyanins with bilitranslocase. Biochem Biophys Res Commun 296, 631-636.

24. McGhie TK \& Walton MC (2007) The bioavailability and absorption of anthocyanins: towards a better understanding. Mol Nutr Food Res 51, 702-713.
25. Maestro A, Terdoslavich M, Vanzo A, et al. (2010) Expression of bilitranslocase in the vascular endothelum and its function as a flavonoid transporter. Cardiovasc Res 85, 175-183.

26. Wu X, Cao G \& Prior RL (2002) Absorption and metabolism of anthocyanins in elderly women after consumption of elderberry or blueberry. J Nutr 132, 1865-1871.

27. Wu X, Pittman HE III \& Prior RL (2004) Pelargonidin is absorbed and metabolized differently than cyanidin after marionberry consumption in pigs. J Nutr 134, 2603-2610.

28. Wu X, Pittman HE III, McKay S, et al. (2005) Aglycones and sugar moieties alter anthocyanin absorption and metabolism after berry consumption in weanling pigs. J Nutr $\mathbf{1 3 5}$ 2417-2424.

29. Wu X, Pittman HE III \& Prior RL (2006) Fate of anthocyanins and antioxidant capacity in contents of the gastrointestinal tract of weanling pigs following black raspberry consumption. J Agric Food Chem 54, 583-589.

30. Matsumoto H, Inaba H, Kishi M, et al. (2001) Orally administered delphinidin 3-rutinoside and cyanidin 3-rutinoside are directly absorbed in rats and humans and appear in the blood as the intact forms. J Agric Food Chem 49, 1546-1551.

31. Matsumoto H, Ito K, Yonekura K, et al. (2007) Enhanced absorption of anthocyanins after oral administration of phytic acid in rats and humans. J Agric Food Chem 55, 2489-2496.

32. Tsuda T, Horio F \& Osawa T (1999) Absorption and metabolism of cyanidin 3-O-beta-D-glucoside in rats. FEBS Lett $\mathbf{4 4 9}$, 179-182.

33. Vitaglione P, Donnarumma G, Napolitano A, et al. (2007) Protocatechuic acid is the major human metabolite of cyanidin-glucosides. J Nutr 137, 2043-2048.

34. Kay CD, Kroon PA \& Cassidy A (2009) The bioactivity of dietary anthocyanins is likely to be mediated by their degradation products. Mol Nutr Food Res 53, Suppl. 1, S92-S101.

35. Woodward G, Kroon P, Cassidy A, et al. (2009) Anthocyanin stability and recovery: implications for the analysis of clinical and experimental samples. J Agric Food Chem 57, 5271-5278.

36. Prior RL \& Wu X (2006) Anthocyanins: structural characteristics that result in unique metabolic patterns and biological activities. Free Radic Res 40, 1014-1028.

37. Cao G, Muccitelli HU, Sanchez-Moreno C, et al. (2001) Anthocyanins are absorbed in glycated forms in elderly women: a pharmacokinetic study. Am J Clin Nutr 73, 920-926.

38. Milbury PE, Cao G, Prior RL, et al. (2002) Bioavailablility of elderberry anthocyanins. Mech Ageing Dev 123, 997-1006.

39. Bitsch I, Janssen M, Netzel M, et al. (2004) Bioavailability of anthocyanidin-3-glycosides following consumption of elderberry extract and blackcurrant juice. Int J Clin Pharmacol Ther 42, 293-300.

40. Bitsch R, Netzel M, Sonntag S, et al. (2004) Urinary excretion of cyanidin glucosides and glucuronides in healthy humans after elderberry juice ingestion. J Biomed Biotechnol 5, 343-345.

41. Kay CD, Mazza G, Holub BJ, et al. (2004) Anthocyanin metabolites in human urine and serum. Br J Nutr $9 \mathbf{1}$ 933-942.

42. Frank T, Janssen M, Netzet G, et al. (2007) Absorption and excretion of elderberry (Sambucus nigra L.) anthocyanins in healthy humans. Methods Find Exp Clin Pharmacol 29, 525-533.

43. Wiczkowski W, Romaszko E \& Piskula MK (2010) Bioavailability of cyanidin glycosides from natural chokeberry (Aronia melanocarpa) juice with dietary-relevant dose of anthocyanins in humans. J Agric Food Chem $\mathbf{5 8}$, $12130-12136$. 